\title{
Vinylogous hydrazone strategy for the organocatalytic alkylation of heteroaromatic derivatives
}

\author{
Beata Łukasik, ${ }^{[a], \ddagger}$ Justyna Kowalska, ${ }^{[a], \ddagger}$ Sebastian Frankowski ${ }^{[a]}$ and Łukasz Albrecht ${ }^{*[a]}$ \\ Dedicated to Professor Tomasz Janecki on the occasion of his $70^{\text {th }}$ birthday.
}
[a] Dr. B. Łukasik, J. Kowalska, S. Frankowski, prof. Ł. Albrecht
Institute of organic chemistry, Faculty of Chemistry
Lodz University of Technology
Żeromskiego 116, 90-924 Łódź (Poland)
$¥$ These authors contributed equally.
E-mail: lukasz.albrecht@p.lodz.pl
Homepage: http://www.a-teamlab.p.lodz.pl/

Supporting information for this article is given via a link at the end of the document.

\begin{abstract}
A new umpolung approach for the asymmetric FriedelCrafts-type alkylation of electron-poor heteroaromatic systems has been developed. It is based on the vinylogous reactivity of hydrazones derived from heteroaromatic aldehydes. Donating effect of the hydrazone moiety can be efficiently transferred over the heteroaromatic system activating it towards asymmetric FriedelCrafts reaction with $\alpha, \beta$-unsaturated aldehydes realized under aminocatalytic conditions. Excellent enantioselectivities have been obtained owing to the application of MacMillan imidazolidinone catalyst. Unmasking of hydrazone moiety has been also realized resulting in the development of a unique strategy for the asymmetric functionalization of electron-poor heteroaromatic systems.
\end{abstract}

The chemistry and biology of heteroaromatic compounds constitutes a fascinating and highly relevant field of research (Scheme 1, top). ${ }^{[1-3]}$ Among various heteroaromatic frameworks, furan and its derivatives occupy a prominent position. ${ }^{[1]}$ They are present in various natural products and bioactive molecules. ${ }^{[4]}$ Therefore, methods for their preparation in a stereoselective fashion are of importance in contemporary organic synthesis. Friedel-Crafts reaction constitutes a powerful method for the functionalization of electron-rich (hetero)aromatic compounds. ${ }^{[5]}$ Surprisingly, the application of furan derivatives in this important reaction is not very common ${ }^{[6]}$ and mostly limited to hydroxylgroup-activated furans in the Mukayama-type reactions. ${ }^{[7]}$ Recently, Chen and co-workers demonstrated that furans can be activated towards Friedel-Crafts reaction via the formation of the corresponding enamine intermediate (Scheme 1, middle). ${ }^{[8]}$ Functionalization of electron-poor heteroaromatic derivatives via Friedel-Crafts reaction is not possible due to decreased nucleophilicity of such systems.

Hydrazones constitute synthetically useful molecules that undergo various annulation reactions, including (3+2)cycloadditions, leading to the synthesis of nitrogen-containing heterocycles. ${ }^{[9]}$ On the other hand, very important application of this group of reactants relates to their ability to induce umpolung of aldehydes inverting their properties from electrophilic to nucleophilic (Scheme 1, bottom). ${ }^{[10]}$ Notably, the nucleophilic properties of hydrazones can be transferred over conjugated double bonds including heteroaromatic systems, thus resulting in the vinylogous reactivity ${ }^{[11]}$ of this class of compounds. ${ }^{[10 a, 12,13]}$
Surprisingly, asymmetric strategies involving vinylogous hydrazone umpolung are missing in the literature.
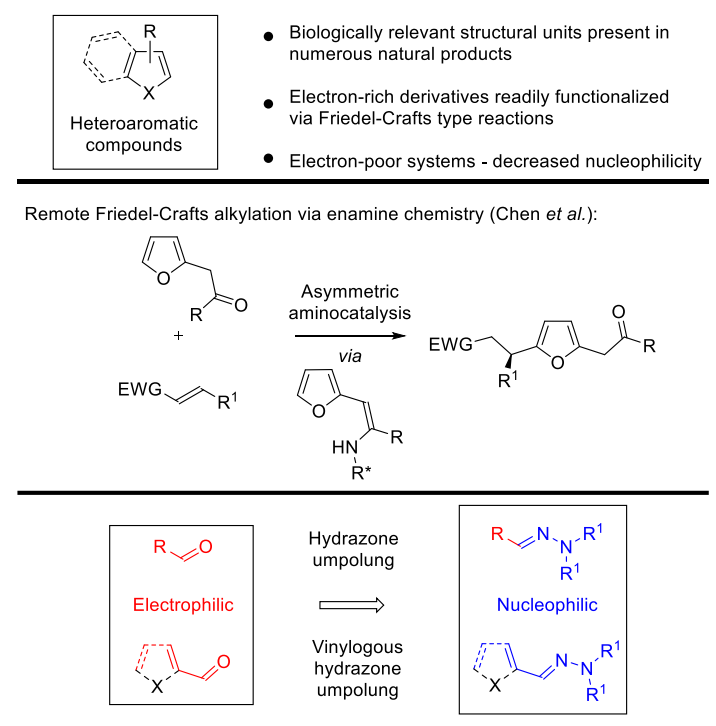

Scheme 1. Importance of heteroaromatic compounds and the principles of hydrazone umpolung.

Given the importance of heteroaromatic compounds in the lifescience industry and the potential of hydrazone in delivering umpolung of aldehydes, the task of the development of a new, asymmetric approach for the electrophilic functionalization of heteroaromatic aldehydes was undertaken (Scheme 2, top). It was envisioned that the corresponding hydrazone, readily available from the aldehyde via condensation with the hydrazine should participate in the Friedel-Crafts reaction with hydrazone donating effect being transferred over heteroaromatic system. Furthermore, it was anticipated that subsequent unmasking of the hydrazone moiety should be possible under mild conditions. Notably, depending on the employed conditions access to various electron-withdrawing groups should be accessible. Within present studies, $\alpha, \beta$-unsaturated aldehydes were selected as model electrophiles due to their ability to undergo various Michael-type 
reactions proceeding under aminocatalytic conditions according to iminium ion activation (Scheme 2, top).

Herein, we present our studies on the application of vinylogous hydrazone strategy for the activation of electron-poor furan derivatives towards the Friedel-Crafts-type reactions. The alkylation of hydrazones 1 with $\alpha, \beta$-unsaturated aldehydes 2 was realized under aminocatalytic conditions providing facile access to aldehydes 5 . The activation strategy proved viable for a wide variety of heteroaromatic systems. The possibility of unmasking the hydrazone moiety was also demonstrated.

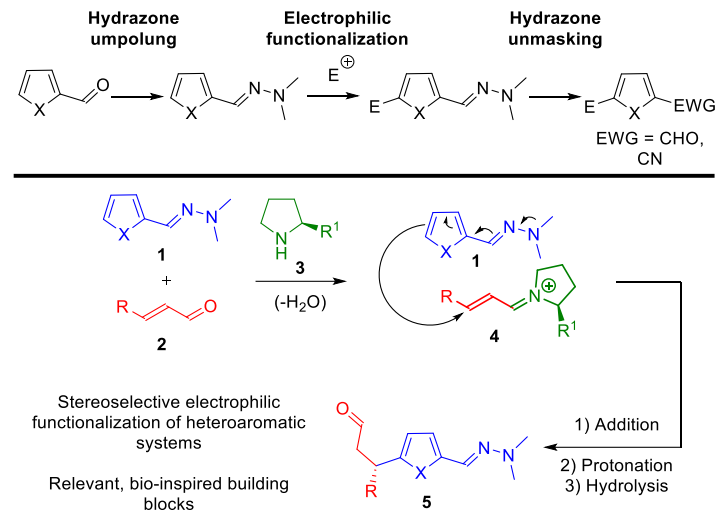

Scheme 2. Vinylogous hydrazone strategy for the asymmetric alkylation of heteroaromatic aldehydes

Optimization studies were performed using $\mathrm{N}, \mathrm{N}$ dimethylhydrazone $\mathbf{1 a}$ derived from 2-furfural and transcinnamaldehyde 2a. Initial experiments were conducted in dichloromethane at ambient temperature with diphenylprolinol trimethylsilyl ether $\mathbf{3 a}$ as catalyst. Disappointingly, under these conditions only traces of the desired product 5 a were obtained (Table 1, entry 1). Delightfully, the presence of acidic additive enhanced the efficiency of the process, leading to significant improvement in conversion of substrates (Table 1, entry 2). Due to the fact that strong acid can promote the hydrazone deprotection, 1.2-fold excess of 1a was used. In the course of further studies, it was found that the change of aminocatalyst 3 had a significant impact on the reaction outcome. Utilization of aminocatalyst $\mathbf{3 b}$ accelerated the transformation rate and the adduct $7 \mathrm{a}$ was isolated after olefination reaction with Wittig reagent 6. Unfortunately, the enantioselectivity of the organocatalytic process was only moderate (Table 1 , entry 3 ). Subsequently, imidazolidinone $3 \mathbf{3 c}$, which previously proved effective in the Friedel-Crafts alkylation of heteroaromatic derivatives, ${ }^{[14]}$ was used, giving access to product 5 a with high conversion after $20 \mathrm{~h}$ at room temperature, however, $7 \mathrm{a}$ was obtained in nearly racemic form (Table 1 , entry 5). To our delight, when the reaction was conducted in the presence of $3 \mathrm{c}$ at $-20^{\circ} \mathrm{C}$, a significant increase of enantioselectivity was observed without loss of reactivity (Table 1, entry 6 ). Further solvent screening indicated toluene as the most suited medium for this transformation (Table 1, entries 6-10). Furthermore, concentration had no noticeable impact on the developed reactivity (Table 1 , entries 11,12 ), thus establishing the final reaction conditions (Table 1 , entry 10 ).
Table 1. Asymmetric alkylation of hydrazones derived from heteroaromatic aldehydes - optimization studies. ${ }^{[a]}$

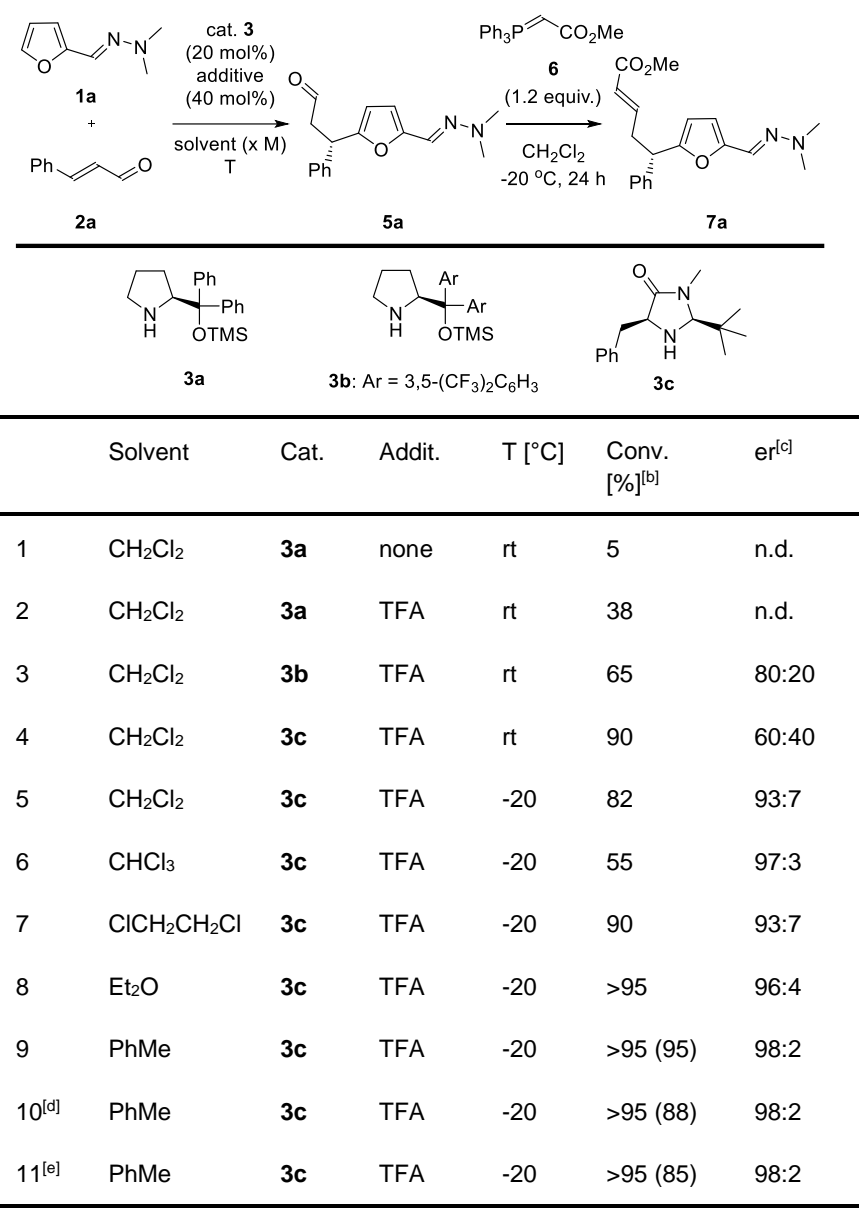

[a] Reactions performed on a $0.05 \mathrm{mmol}$ scale using $\mathbf{1 a}$ (1.2 equiv) and $\mathbf{2 a}$ (1.0 equiv.) in $0.2 \mathrm{~mL}$ of the solvent for $20 \mathrm{~h}$ (for full screening account, see Supporting Information). [b] Conversion as determined by ${ }^{1} \mathrm{H}$ NMR of a crude reaction mixture. Isolated yield is given in parentheses. [c] Determined by a chiral stationary phase UPC ${ }^{2}$. [d] Reaction performed in $0.1 \mathrm{~mL}$ of the solvent [e] Reaction performed in $0.4 \mathrm{~mL}$ of the solvent.

With the optimal reaction conditions established (Table 1, entry $10)$, the substrate scope of the reaction was explored both with respect to aldehydes 2 (Table 2) and hydrazones 1 (Scheme 3). In order to facilitate the isolation and determination of enantiomeric ratios, obtained adducts 5 were directly converted into the corresponding esters 7 using Wittig reagent $\mathbf{6}$ (isolation of aldehydes $\mathbf{5}$ proved also possible. For details, see Supporting Information). In the first step of the scope study, various $\alpha, \beta$ unsaturated aldehydes 2 were reacted with hydrazone $\mathbf{1 a}$ under optimized conditions. Initially, cinnamaldehydes bearing substituent at different positions and with different electronic properties were investigated (Table 2, entries 2-6). To our delight, all reactions proceeded smoothly affording target products $\mathbf{7}$ in high yields and enantioselectivities. When orthomethoxycinnamaldehyde 2d was applied, a slightly lower enantioselectivity was observed (Table 2 , entry 4 ), but the yield remained excellent. The use of 2 -furyl-substituted $\alpha, \beta$ unsaturated aldehyde $\mathbf{2} \mathbf{g}$ also resulted in the drop of enantioselectivity (Table 2 , entry 7 ). In the case of the 1-naphthyl group bearing $\mathbf{2 h}$, high enantioselectivity and yield was maintained (Table 2, entry 8 ). Furthermore, the possibility to 
introduce various aliphatic substituents in the $\beta$ position of the aldehydes 2 was investigated. It was found that regardless the length of aliphatic chain in $\mathbf{2}$, expected products $\mathbf{7}$ were obtained in high yields and enantioselectivities (Table 2, entries 9,10). Aldehydes $\mathbf{2 k}$, I bearing functional groups in their side chain turned out to be excellent electrophiles and the desired products $6 \mathbf{k}, \mathbf{I}$ were obtained in very good yields and in a highly enantioselective fashion (Table 2, entries 11,12).

Table 2. Asymmetric alkylation of hydrazones derived from heteroaromatic aldehydes - scope of $\alpha, \beta$-unsaturated aldehydes 2 . $^{[\mathrm{a}]}$

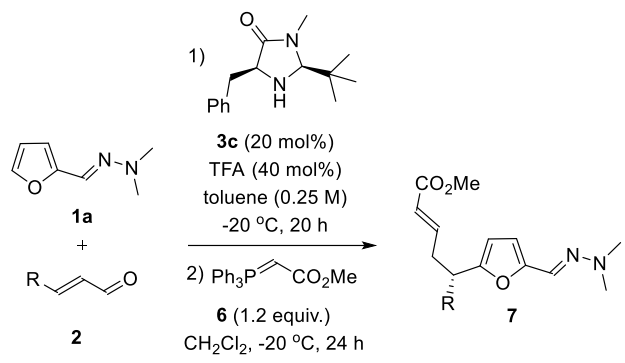

\begin{tabular}{|c|c|c|c|c|}
\hline Entry & $\mathrm{R}$ & Yield [\%] & $E / Z^{[\mathrm{b}]}$ & $\mathrm{er}^{[\mathrm{c}]}$ \\
\hline 1 & $\mathrm{Ph}$ & 95 & $>20: 1$ & $98: 2$ \\
\hline 2 & 4- $\mathrm{MeOC}_{6} \mathrm{H}_{4}$ & 88 & $>20: 1$ & $94.5: 5.5$ \\
\hline 3 & 3- $\mathrm{MeOC}_{6} \mathrm{H}_{4}$ & 85 & $>20: 1$ & $97: 3$ \\
\hline $4^{[\mathrm{d}]}$ & $2-\mathrm{MeOC}_{6} \mathrm{H}_{4}$ & 95 & $>20: 1$ & $91: 9$ \\
\hline 5 & $4-\mathrm{ClC}_{6} \mathrm{H}_{4}$ & 97 & $>20: 1$ & $98: 2$ \\
\hline 6 & $4-\mathrm{NO}_{2} \mathrm{C}_{6} \mathrm{H}_{4}$ & 93 & $>20: 1$ & $99: 1$ \\
\hline 7 & 2-Furyl & 85 & $>20: 1$ & $91: 9$ \\
\hline 8 & 1-Naphthyl & 92 & $18: 1$ & $96: 4$ \\
\hline 9 & $\mathrm{Me}$ & 85 & $>20: 1$ & $96: 4$ \\
\hline 10 & $n \mathrm{Pr}$ & 81 & $>20: 1$ & $98: 2$ \\
\hline 11 & $\mathrm{BnOCH}_{2}$ & 91 & $>20: 1$ & $97: 3$ \\
\hline 12 & Z-3-Hexenyl & 96 & $>20: 1$ & $98: 2$ \\
\hline
\end{tabular}

[a] Reactions performed on a $0.1 \mathrm{mmol}$ scale using $\mathbf{1 a}$ (1.2 equiv) and 2 (1.2 equiv.) in $0.4 \mathrm{~mL}$ of the solvent for $20 \mathrm{~h}$. [b] Determined by ${ }^{1} \mathrm{H}$ NMR of a crude reaction mixture after Wittig olefination. [c] Determined by a chiral stationary phase UPC ${ }^{2}$. [d] Wittig olefination performed at room temperature.

Further studies were focused on the application of various hydrazones 1 in the reaction with cinnamaldehyde 2a (Scheme $3)$. Notably, in this part of scope studies final reaction parameters had to be fine-tuned in some of the cases in order to obtain higher reactivity or enantioselectivity. When furan $\mathbf{1} \mathbf{b}$ bearing hydrazone moiety at the position 3 was utilized, the reaction proceeded efficiently, but a drop of enantioselectivity was noted (Scheme 3, compound $\mathbf{7 m}$ ). In case of pyrrole- as well as thiophene-derived nucleophiles 1b and 1c, excellent enantioselectivities were obtained (Scheme 3, compounds $\mathbf{7 n}, \mathbf{0}$ ). It was also found that the activating effect of the hydrazone moiety can be transferred through more than just two double bonds as it was observed in the case of simple heteroaromatic derivatives 1a-c. To our delight, thienothiophene derivative $1 \mathbf{d}$ reacted smoothly (transfer of the hydrazone effect over 3 double bonds) providing $7 p$ with excellent enantioselectivity (Scheme 3, compound 7p). Similarly, hydrazone $1 \mathbf{e}$ derived from the corresponding $\alpha, \beta$-unsaturated aldehyde $\mathbf{2 g}$ proved also viable substrate for our transformation with the alkylation taking place preferentially at the remote furan ring (Scheme 3, compound 7q).

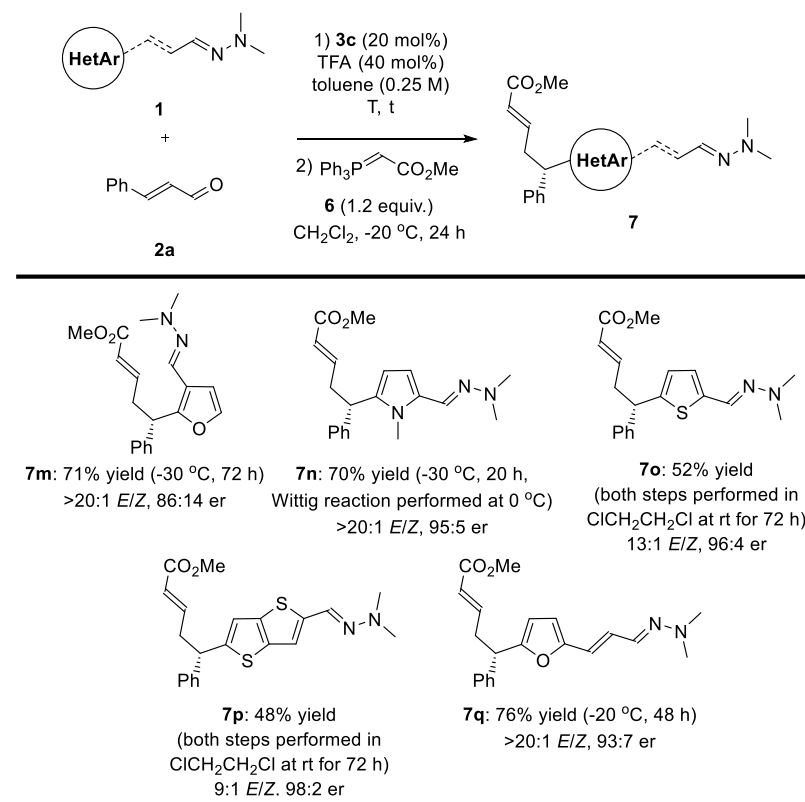

Scheme 3. Asymmetric alkylation of hydrazones derived from heteroaromatic aldehydes - scope of hydrazones 1 .

In the course of further studies, the possibility to unmask hydrazone moiety in 7a was demonstrated (Scheme 4). Oxidation of compounds bearing carbon=nitrogen double bond constitutes a useful method for the preparation of nitriles and related products. ${ }^{[15]}$ Therefore, $7 \mathrm{a}$ was subjected to the oxidation reaction with $m$-CPBA (Scheme 4 , top). The reaction was carried out in dichloromethane at room temperature for 4 hours affording the corresponding nitrile $\mathbf{8 a}$ in $95 \%$ yield. Furthermore, protocol for the deprotection of the aldehyde moiety was also developed. The reaction was realized under mild conditions to provide aldehyde 9a in $85 \%$ yield. Notably, both transformations proceeded with preservation of optical purity introduced at the organocatalytic step as both $\mathbf{8 a}$ and $\mathbf{9 a}$ were obtained in a highly enantiomerically enriched form.

The absolute configuration of the product $\mathbf{7 n}$ was assigned by single crystal $\mathrm{X}$-ray analysis (for details, see Supporting Information). ${ }^{[16]}$ The stereochemistry of remaining products was assigned by analogy. Notably, such a stereochemical reaction outcome is in accordance with classical aminocatalytic cycle proceeding via iminium ion activation using imidazolidinone $3 \mathrm{c}$ as catalysts. ${ }^{[14]}$ 


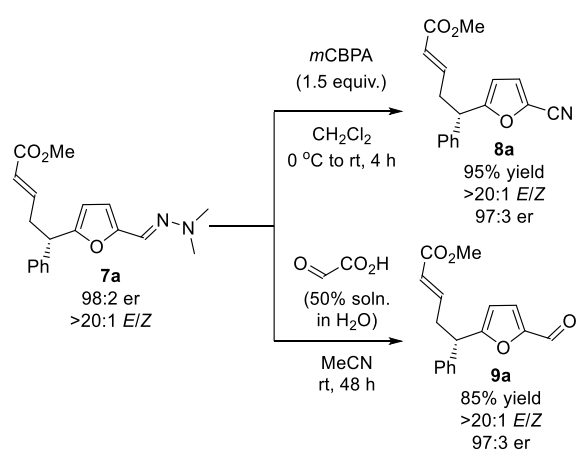

Scheme 4. Asymmetric alkylation of hydrazones derived from heteroaromatic aldehydes - unmasking of hydrazone 1 .

In conclusion, we developed a unique strategy for the asymmetric functionalization of electron-poor heteroaromatic derivatives. It utilized the application of hydrazones derived from heteroaromatic aldehydes as key reactants. It was found that the donating effect of hydrazone moiety can be efficiently transfered over two or three double bonds that were a part of aromatic systems providing a useful tool for the asymmetric alkylation of heteroaromatic moiety. High stereoselectivity was obtained by the use of imidazolidinone catalyst. The possibility to transform hydrazone group into formyl or nitrile moieties was also demonstrated

\section{Acknowledgements}

This project was realized within the Sheng programme (grant number: UMO-2018/30/Q/ST5/00466) from the National Science Centre, Poland. Thanks are expressed to Dr. Lesław Sieroń (Faculty of Chemistry, Lodz University of Technology) for performing X-ray analysis.

Keywords: organocatalysis $\cdot$ aminocatalysis $\cdot$ umpolung • Friedel-Crafts reaction $\bullet$ hydrazones

[1] a) B. A. Keay, P. W. Dibble in Comprehensive Heterocyclic Chemistry II, Vol. 2 (Eds.: A. R. Katritzky, C. W. Rees, E. F. Schriven, C.W. Bird), Pergamon, Oxford, 1996, pp. 395; b) J. B. Sperry, D. L. Wright, Curr. Opin. Drug Discovery Devel. 2005, 8, 723-740.

[2] a) V. Sharma, P. Kumar, D. Pathak, J. Heterocycl. Chem. 2010, 47, 491 502; b) W. Gul, M. T. Hamann, Life Sci. 2005, 78, 442-453.

[3] a) T. Lindel, Alkaloids: Chemistry and Biology 2017, 77, 117-219; b) V. Bhardwaj, D. Gumber, V. Abbot, S. Dhimana, P. Sharma, RSC Adv. 2015, 5, 15233-15266

[4] a) A. Boto, L. Alvarez, in: Heterocycles in Natural Product Synthesis, (Eds.: K. Majumdar, S. K. Chattopadhyay), Wiley-VCH, Weinheim, 2011; b) S. F. Kirsch, Org. Biomol. Chem. 2006, 4, 2076-2080.

[5] For general reviews on Friedel-Crafts reactions, see: a) N. O. Calloway, Chem. Rev. 1935, 17, 327-392; b) T. B. Poulsen, K. A. Jørgensen, Chem. Rev. 2008, 108, 2903-2915; c) M. Rueping, B. J. Nachtsheim, Beilstein J. Org. Chem. 2010, 6, 1-24; for reviews on organocatalytic Friedel-Crafts reactions, see: d) S.-L. You, Q. Cai, M. Zeng, Chem. Soc Rev. 2009,38, 2190-2201; e) M. M. Heravi, V. Zadsirjan, M. Heydari, B. Masoumi, Chem. Rec. 2019,19, 2236-2340.
[6] For the reports of organocatalytic Friedel-Crafts alkylation of 2 methoxyfuran, see: a) D. Uraguchi, K. Sorimachi, M. Terada, J. Am Chem. Soc. 2004, 126, 11804-11805; b) A. Kondoh, Y. Ota, T. Komuro, F. Egawa, K. Kanomatab, M. Terada, Chem. Sci. 2016, 7, 1057-1062 c) M. Hatano, H. Okamoto, T. Kawakami, K. Toh, H. Nakatsuji, A Sakakura, K. Ishihara, Chem. Sci. 2018, 9, 6361-6367.

[7] For reviews on Mukayama-type chemistry, see: a) G. Casiraghi, F. Zanardi, L. Battistini, G. Rassu, Synlett, 2009, 1525-1542; b) B. Mao, M. Fañanás-Mastral, B. L. Feringa, Chem. Rev. 2017, 117, 10502-10566; c) A. R. Choudhury, S. Mukherjee, Chem. Soc. Rev. 2020, 49, 67556788

[8] a) J.-L. Li, C.-Z. Yue, P.-Q. Chen, Y.-C. Xiao, Y.-C. Chen, Angew. Chem Int. Ed. 2014, 53, 5449; b) G.-J. Yang, W. Du, Y.-C. Chen, J. Org. Chem. 2016, 81, 10056-10061; for a related approach, see: c) X.-R. Zhang, S. L. Zhou, Y. Yuan, W. Du, Y.-C. Chen, Synlett 2017, 28, 1771-1774.

[9] For a review, see: N. P. Belskaya, A. I. Eliseeva, V. A. Bakulev, Russ Chem. Rev. 2015, 84, 1226-1257.

[10] For reviews on the hydrazone umpolung reactivity, see: a) R. Brehme D. Enders, R. Fernandez, J. M. Lassaletta, Eur. J. Org. Chem. 2007 2007, 5629-5660; b) M. de Gracia Retamosa, E. Matador, D. Monge, J. M. Lassaletta, R. Fernández, Chem. Eur. J. 2016, 22, 13430-13445; For the recent utilization of hydrazone umpolung in organocatalytic approaches: c) K. Nagaraju, R. Gurubrahamam, K. Chen, J. Org. Chem. 2020, 85, 7060-7067; d) E. Matador, M. de Gracia Retamosa, D. Monge, R. Fernández, J. M. Lassaletta, Chem. Commun., 2020,56, 9256-9267; e) M. Gómez-Martínez, M. del Carmen Pérez-Aguilar, D. G. Piekarski, C G. Daniliuc, O. García Mancheño, Angew. Chem. Int. Ed. 2021, 60, 5096-5101.

[11] C. Curti, L. Battistini, A. Sartori, F. Zanardi, Chem. Rev. 2020, 120, 2448 2612

[12] For recent reports on vinylogous hydrazone umpolung, see: a) P. BernalAlbert, H. Faustino, A. Gimeno, G. Asensio, J. L. Mascareñas, F. López, Org. Lett. 2014, 16, 6196-6199; b) Y. Hashimoto, R. Abe, N. Morita, O. Tamura, Org. Biomol. Chem. 2018,16, 8913-8916; c) Y. Hashimoto, T. Ikeda, A. Ida, N. Morita, O. Tamura, Org. Lett. 2019, 21, 4245-4249.

[13] a) P. V. Khodakovskiy,P. K. Mykhailiuk, D. M. Volochnyuka, A. A Tolmachev, Synthesis 2010, 6, 979-984; b) J. Benites, J. A. Valderrama F. Rivera. L. Rojo, N. Campos, M. Pedro, M. S. José Nascimento, Bioorg. Med. Chem. 2008, 16, 862-868.

[14] a) G. Lelais, D. W. C. MacMillan, Aldrichimica Acta, 2006, 39, 79-87; b) R. Mahrwald, in Comprehensive Enantioselective Organocatalysis, (P. I. Dalko Ed.) 2013, 69-95; c) D. Seebach, U. Grošelj, D. M. Badine, W. B. Schweizer, A. K. Beck, Helv. Chim. Acta, 2008, 91, 1999-2034.

[15] a) J. Młochowski, S. B. Said, Pol. J. Chem., 1992, 66, 1901-1928; b) D. Christensen, K. A. Jørgensen, J. Org. Chem., 1989, 54, 126-131; c) J. Larsen, K. A. Jørgensen, D. Christensen, J. Chem. Soc. Perkin Trans., 1991, 1, 1187-1190.

[16] CCDC 2064989 (7n) contains the supplementary crystallographic data for this paper. These data can be obtained free of charge from The Cambridge Crystallographic Data Centre via www.ccdc.cam.ac.uk/structures. 


\section{Entry for the Table of Contents}

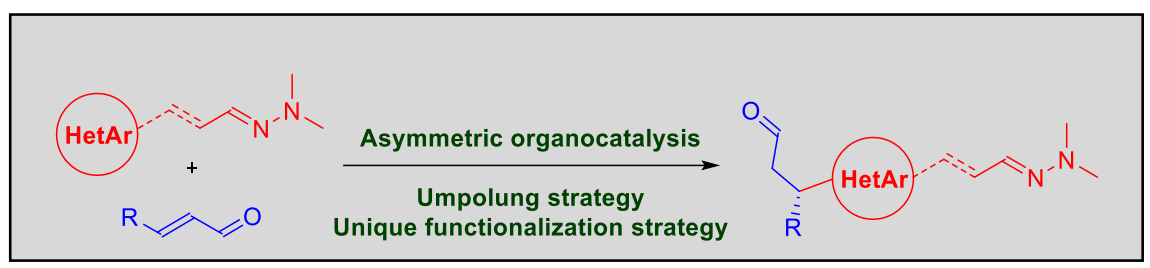

A unique functionalization strategy of electron-poor heteroaromatic derivatives has been developed. It is based on the vinylogous reactivity of hydrazones derived from heteroaromatic aldehydes. The donating effect of hydrazone moiety is efficiently transfer over the heteroaromatic system. The highly enantioselective asymmetric alkylation with $\alpha, \beta$-unsaturated aldehydes thus developed was realized in the presence of chiral imidazolidinone aminocatalyst.

Institute and/or researcher Twitter usernames: @a_teamlab 